\title{
The Low-Loss Spectrum of Individual Carbon Nanotubes Revisited at High Energy Resolution in Real and Momentum Space
}

FS Hage ${ }^{1}$ and QM Ramasse ${ }^{1}$

1. SuperSTEM Laboratory, SciTech Daresbury Campus, Daresbury WA4 4AD, UK

Over the last quarter of a century, carbon nanotubes (CNTs) have been the focus of considerable research efforts in the wider scientific community. During this time, Electron Energy Loss Spectroscopy (EELS) has been used to characterize the plasmonic [1-4] and the electronic [1,3] response of CNTs with great success, utilizing either a purpose-built stand-alone spectrometer apparatus [1-3] or a spectrometer attached to a Scanning Transmission Electron Microscope (STEM) [4]. While the spatial resolution of STEM-EELS far exceeds that of stand-alone spectrometers, STEM-EELS experiments have until recently been limited by a poorer energy resolution. In exploiting the novel capabilities of the monochromated Nion UltraSTEM100MC dedicated STEM [5] it is now possible to study the plasmonic and electronic response of single CNTs at high spatial resolution, while providing spectral information comparable to that of a dedicated spectrometer. In the present work, measurements at high spatial resolution allowing a direct correlation of spectral features with the atomic-scale structure of CNTs are complemented by measurements resolved in momentum space, a unique combination which provides a comprehensive analysis of the low-loss spectrum of individual free-standing CNTs.

Figure 1a shows a Medium Angle Annular Dark Field (MAADF) image of a single-wall CNT. Spectra acquired from the center of the tube (1) and at a position $\sim 3 \mathrm{~nm}$ from the tube center (2) are shown in Figure $1 \mathrm{~b}$. The spectra are characterised by the presence of a number of sharp peaks attributed to socalled van Hove singularities (VHSs, $<\sim 5 \mathrm{eV})[1,3]$, followed by peaks assigned to the $\pi(\sim 5 \mathrm{eV})$ and the $\pi+\sigma$ plasmons $(\sim 15 \mathrm{eV})$ [1-3]. The energy losses at which these "VHS peaks" occur are reported to be characteristic of the separation of VHSs in the CNT electronic density of states [1, 3] and thus essential in determining their electronic response for practical applications. An observed red-shift of the plasmon peaks when going from a penetrating (1) to an aloof (2) beam geometry is also consistent with literature [4]. Note the lack of a comparable red-shift for the VHS peaks.

Spectra acquired in the optical limit $\left(\mathrm{q} \rightarrow 0.0 \AA^{-1}\right)$ are compared for 1,2 and 3 walled CNTs in Figure $2 \mathrm{a}$. All three spectra show a greatly varying number of VHS peaks. In addition to confirming the overall plasmon peak shapes previously reported for single tubes [4], the present data exhibit an apparent fine structure of both plasmon peaks varying significantly from tube to tube. These variations hint at very different electronic configurations due to the CNTs' structure.

Furthermore, resolving EEL spectra in momentum space allows for access to information not readily available from conventional high spatial resolution EELS. Figure $2 \mathrm{~b}$ shows the momentum-resolved spectra for the single-wall CNT depicted in Figure 1a. The "VHS peaks" (see black arrows) at $\approx 2.1 \mathrm{eV}$ and $\approx 3.5 \mathrm{eV}$ are non-dispersive (no peak shift with increasing momentum transfer $\hbar \mathrm{q}$ ), consistent with literature measurements on a "bulk" sample of purified single wall CNTs [1]. In addition, the spectra in Figure $1 b$ reveal the splitting of the " $\pi$ plasmon peak" into two distinct plasmons $\pi_{1}$ and $\pi_{2}$ (see red arrow). The $\pi_{1}$ peak is non-dispersive $(\approx 4.8 \mathrm{eV})$ thus indicating confinement perpendicular to the CNT axis $[2,3]$, while the $\pi_{2}$ peak is dispersive $\left(\approx 5.7-6.0 \mathrm{eV}\right.$ for $\left.\mathrm{q}=0.0-0.24 \AA^{-1}\right)$ which in turn indicates significant plasmon propagation along the CNT axis $[2,3,6]$. 
References:

[1] T Pichler et al., PRL 80 (1998), p. 4729.

[2] C Kramberger et al., PRL 100 (2008), 196803.

[3] C Kramberger et al., Nanotechnology 24 (2013), 405202.

[4] M Kociak et al., PRB 61 (2000) 13936, BW Reed and M Sarikaya, PRB 64 (2001), 195404, O Stéphan et al., PRB 66 (2002) 155422.

[5] OL Krivanek et al., Phil. Trans. Roy. Soc. 367 (2009), p. 3683

[6] SuperSTEM is the UK National Facility for aberration-corrected STEM and is funded by the UK Engineering and Physical Sciences Research Council (EPSRC). N Dellby and TC Lovejoy (Nion Company, WA, USA) are thanked for useful discussions and advice. U Bangert (University of Limerick, Ireland) is thanked for providing CNT samples.
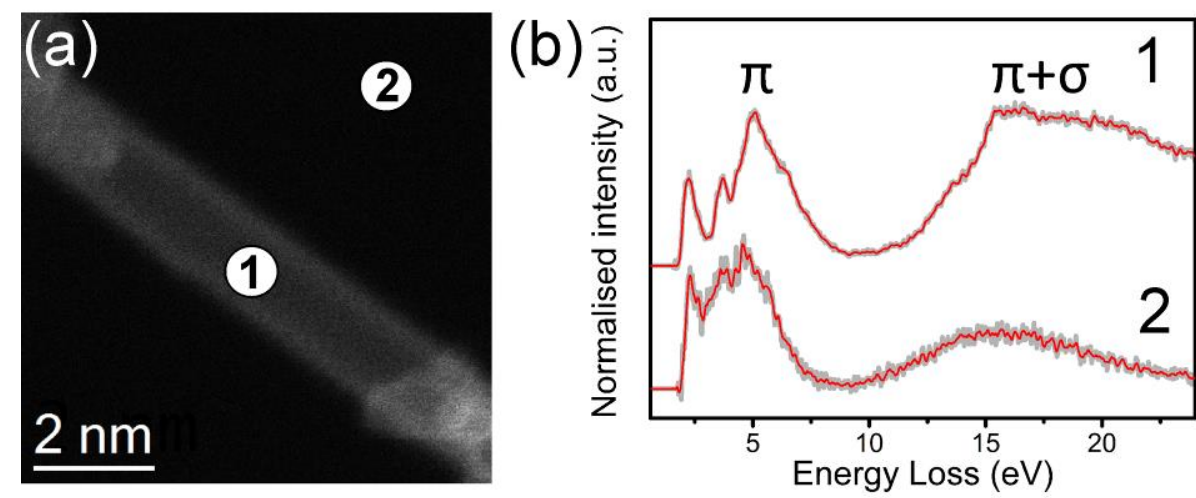

Figure 1. (a) MAADF image of a single wall carbon nanotube. (b) ZLP subtracted spectra acquired from positions 1 and 2 in (a). Smoothed spectra (red solid lines) are overlaid all original data (grey solid lines) as a guide to the eye.
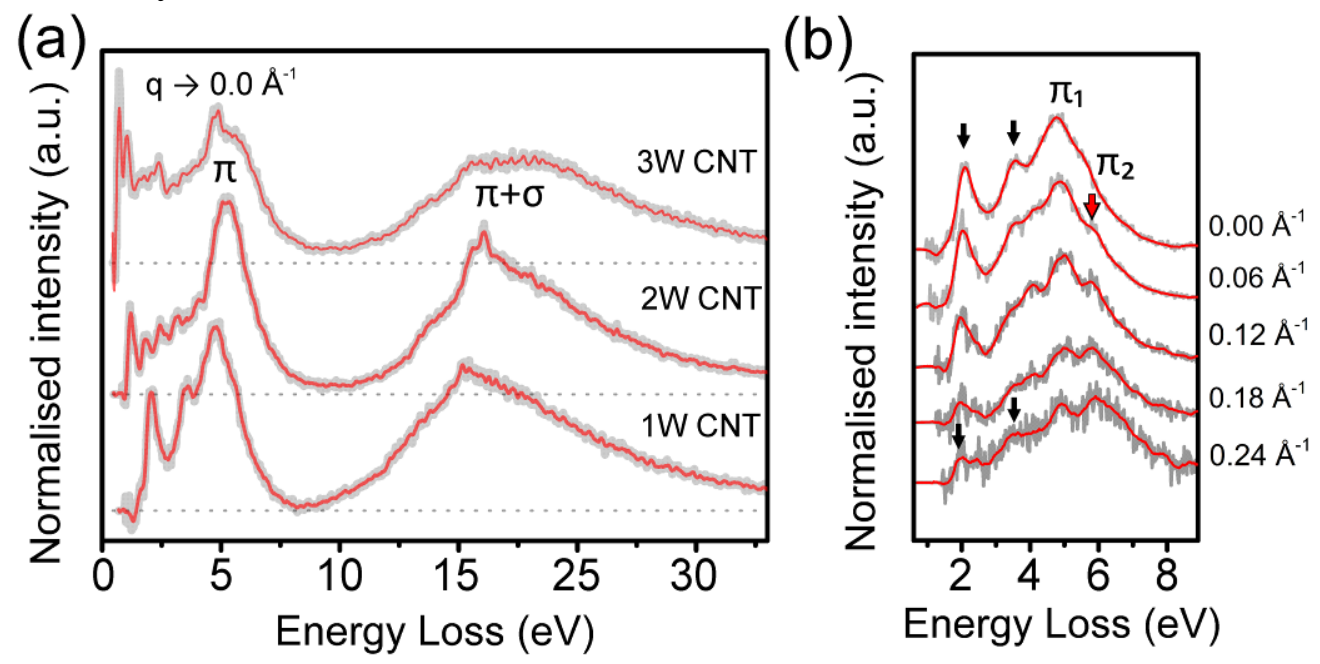

Figure 2. (a) Examples of momentum resolved (ZLP subtracted) loss spectra of single free-standing 1, 2 and 3 wall CNTs (in the optical limit: $\mathrm{q} \rightarrow 0.0 \AA^{-1}$ ). (b) Momentum resolved spectra from the single wall tube in Figure 1. Smoothed spectra (red solid lines) are overlaid all original data (grey solid lines) as a guide to the eye. The dotted lines in (a) indicate zero intensity for each spectrum. 